\title{
Developing a Tool to Cost Gaps in Implementation of IHR (2005) Core Capacities
}

\author{
Stella Chungong ${ }^{1}$, Jun Xing ${ }^{1}$, Rajesh Sreedharan ${ }^{1}$, Stephane de La Rocque ${ }^{1}$, Rebecca \\ $\mathrm{Katz}^{2}$, Julie E. Fischer ${ }^{2}$, Mahomed Patel ${ }^{3}$, Lisa D. Ferland ${ }^{* 4}$, Meeyoung Park ${ }^{4}$, Ngozi \\ Erondu ${ }^{4}$, William MacWright ${ }^{4}$ and Scott J. McNabb ${ }^{4}$
}

${ }^{1}$ World Health Organization, Geneva, Switzerland; ${ }^{2}$ George Washington University, Washington, DC, DC, USA; ${ }^{3}$ Australian National

University, Canberra, ACT, Australia; ${ }^{4}$ Emory University, Atlanta, GA, USA

\section{Objective}

To support national decision makers in estimating the costs for closing identified gaps in meeting the national core capacity building requirements of the International Health Regulations (IHR [2005]), including start-up and operating costs.

\section{Introduction}

The International Health Regulations were revised in 2005 to adapt to increasing diversity of emerging health threats, globalization, changing trends in trade and travel, and the need for collective effort to address the international spread of disease.

States Parties to the Regulations have committed to meeting their obligations within a certain timeframe, including achieving the minimum IHR core capacities throughout their territories. By May 2013, over 100 WHO Member States (MS) reported not having met their national IHR core capacity requirements. Many MS need support in making realistic estimates of their activities, plans and associated costs in a standardized way to support planning and advocacy for building capacity to meet IHR (2005) requirements.

\section{Methods}

WHO, in collaboration with George Washington, Emory, and Australian National Universities, developed a costing tool using a dual end-user interface approach organized by (1) IHR (2005) Core Capacities and (2) public health core functions. Estimated costs are calculated using a template to identify inputs (e.g., personnel compensation, travel and per diem, meeting costs) that can be systematically applied to appropriate public health actions necessary to fulfill each IHR attribute with appropriate multipliers (e.g., number of districts). Actions and inputs are customizable, and specific costs and multipliers are informed by standardized data sets and country responses; these will be further refined by pilot testing.

\section{Results}

The tool will provide users with estimated annualized costs for developing and sustaining public health activities relevant to IHR (2005) implementation. WHO is identifying MS to pilot test the costing tool in Fall 2013 and validate the actions, inputs, and costs, plus usability and utility. Full deployment is targeted for early 2014.

\section{Conclusions}

Identifying actions and costs associated with IHR (2005) implementation is a complex process requiring a thorough understanding of public health workflow processes that occur at the national, intermediate, and local levels. Providing target users, such as National Focal Points, with costs estimates and generalized plans of action facilitates the allocation of funds and development of IHR capacities and public health functions. Furthermore, a tool that can estimate one-time and recurring program costs is an important need for the global public health community.

\section{Keywords}

International Health Regulations; surveillance; economics

\section{References}

1. World Health Organization, World Health Assembly. International Health Regulations (2005). 2nd ed. Geneva: The Organization; 2008.

2. World Health Organization. IHR (2005) Monitoring Framework: checklist and indicators for monitoring progress in the development of IHR core capacities in states parties. Geneva: The Organization; 2010.

3. World Health Organization. Protocol for assessing national surveillance and response capacities for the International Health Regulations (2005). Geneva: The Organization; 2010.

4. Katz R, Haté V, Kornblet S, Fischer JE. Costing framework for International Health Regulations (2005). Emerg Infect Dis [serial on the Internet]. 2012 Jul [22 Aug 2013]. http://dx.doi.org/10.3201/ eid1807.120191

\section{*Lisa D. Ferland}

E-mail: Iferland@publichealthpractice.com 